Annuaire suisse de politique de développement

$10 \mid 1991$

Annuaire Suisse - Tiers Monde 1991

\title{
La Suisse et le groupe de la Banque mondiale
}

Jacques Forster

\section{OpenEdition}

\section{Journals}

Édition électronique

URL : http://journals.openedition.org/aspd/1379

DOI : $10.4000 /$ aspd. 1379

ISSN : 1663-9669

\section{Éditeur}

Institut de hautes études internationales et du développement

\section{Édition imprimée}

Date de publication : 1 janvier 1991

Pagination : 183-205

ISSN : 1660-5934

\section{Référence électronique}

Jacques Forster, "La Suisse et le groupe de la Banque mondiale », Annuaire suisse de politique de développement [En ligne], 10 | 1991, mis en ligne le 13 avril 2013, consulté le 08 septembre 2020. URL : http://journals.openedition.org/aspd/1379 ; DOI : https://doi.org/10.4000/aspd.1379 


\title{
La Suisse ef le groupe de la Banque mondiale
}

\author{
Jacques Forster
}

\section{Introduction}

La question de l'adhésion de la Suisse aux institutions de Bretton Woods ne se pose pas dans les mêmes termes pour le FMI et pour la Banque mondiale ${ }^{1}$; il y a deux raisons à cela:

- en premier lieu l'adhésion au FMI est une condition sine qua non pour devenir membre de la Banque mondiale; face à la question de l'adhésion, les deux institutions ne sont donc pas sur le même pied;

- il faut ensuite relever que depuis 1947, date à laquelle cette question s'est posée pour la première fois aux autorités fédérales, les objections à une adhésion, qui ont varié avec le temps, concernent essentiellement le FMP².

1. Dans le texte qui suit nous emploierons le terme de 'Banque mondiale' dans le même sens que le fait l'institution elle-même: 'L'expression 'la Banque mondiale'(...) désigne à la fois la Banque internationale pour la reconstruction et le développement (BIRD) et son institution affiliée, l'Association internationale de développement (IDA). La BIRD a deux autres filiales, la Société financière internationale (SFI) et l'Agence multilatérale de garantie des investissements (AMGI). Ensemble, ces trois institutions constituent ce qu'on appelle parfois le 'Groupe de la Banque mondiale'. "(Banque mondiale, Rapport 1989, p.3).

2. Voir: - Richard Gerster, Fallstricke der Verschuldung, Z-Verlag, Basel 1982, p.212 ss. - Daniel Kaeser, "Les relations de la Suisse avec les institutions de Bretton Woods", La Vie économique, 2/89, p. 9. - Pierre Languetin, "La Suisse face aux institutions de Bretton Woods", Annuaire Suisse-Tiers Monde, no 10, 1991. 
Aujourd'hui encore, les milieux les plus réservés à l'égard de l'adhésion ne mentionnent guère la Banque mondiale dans leurs prises de position ${ }^{3}$. En fait la possibilité de devenir membre de la Banque mondiale peut être un argument de politique économique extérieure en faveur de l'adhésion au FMI dans la mesure où l'économie suisse tire des avantages assez importants de ses relations avec elle. Sur le plan de la politique de développement, un contre-argument parfois avancé est que la participation à part entière aux activités de la Banque mondiale risque de se faire au détriment de la coopération bilatérale au développement ${ }^{4}$.

On peut donc considérer dans le contexte actuel que l'adhésion à la Banque serait le cas échéant un "sous-produit" largement accepté en Suisse d'une décision d'adhésion au FMI. II n'en reste pas moins que nos relations avec le Groupe de la Banque mondiale sont à la fois anciennes et importantes et qu'elles concernent l'ensemble des acteurs politiques et économiques suisses: en effet les entreprises exportatrices de biens et de services, les banques, les oeuvres d'entraide sont, aux côtés de l'administration fédérale, directement concernées par la nature et l'ampleur de la collaboration spéciale qui s'est développée au fil des ans entre la Suisse et cette institution.

Dans les paragraphes qui suivent, nous évoquerons d'abord l'histoire des relations de la Suisse avec le Groupe de la Banque mondiale avant de présenter les relations qui se sont établies avec chacune des composantes de ce groupe et en particulier avec I'IDA. L'accent mis sur cette dernière institution s'explique par le fait qu'elle est le principal partenaire de la Confédération au sein du Groupe de la Banque mondiale et que c'est par son intermédiaire qu'une part de l'APD de la Suisse est acheminée vers les pays à faible revenu. Nous discuterons enfin dans notre conclusion certains des effets qu'auraient pour la Suisse une pleine participation à la Banque mondiale par rapport au statut très particulier dont elle jouit actuellement.

\section{Historique}

En 1947, lorsqu'il examine pour la première fois la question des relations de la Suisse avec les institutions de Bretton Woods, le Conseil fédéral considère qu'il serait souhaitable que la Suisse puisse adhérer à la BIRD. Ceci n'étant pas possible sans une adhésion préalable au FMI, les autorités suisses cherchent à établir avec la BIRD, et plus tard avec le groupe de la Banque mondiale, des relations répondant à une stratégie de participation maximale sans adhésion. Ceci

3. Il est par exemple intéressant de noter que la prise de position de la Déclaration de Berne de mars 1990 (Solidaire, avril 1990, no 103, p.6) ne mentionne pas une seule fois le nom de la Banque mondiale.

4. Voir Richard Gerster, op.cit.,p. 227. 
se manifeste tout d'abord par la conclusion en 1951, d'un accord facilitant l'accès de la BIRD au marché suisse des capitaux en prévoyant notamment certains allégements fiscaux pour ses emprunts. En 1956 et 1961, la Confédération accorde à la BIRD deux prêts (200 millions de francs à 3,37\% et de 100 millions de francs à 3,75\%). Cet accord et ces prêts font que les entreprises suisses seront autorisées à participer aux adjudications de biens et de services financés par la Banque mondiale. La Suisse partage avec Taiwan (province de Chine) le privilège de pouvoir jouir de cet avantage sans être membre de la Banque mondiale.

Dans les années 1960 et 1970, les relations se développent entre la BIRD et l'économie suisse: avec les banques d'abord qui gèrent ses emprunts de plus en plus importants sur le marché suisse des capitaux; avec les entreprises suisses ensuite dont les ventes dans les pays en développement financées par les crédits de la BIRD acquièrent une importance croissante. Aussi, lorsque l'IDA procède en 1969 à la deuxième reconstitution de ses ressources, la Suisse décide-t-elle d'y participer par un prêt sans intérêt et remboursable en 50 ans de 52 millions de francs. Il devenait en effet difficile pour la Suisse de continuer à bénéficier des activités de la Banque sans participer aux côtés des autres pays industrialisés au financement de l'IDA. La méme opération pour un montant de 130 millions de francs sera répétée en 1972 au titre de la troisième reconstitution.

En 1974, un nouveau pas est franchi dans la coopération lorsque la Suisse est admise en qualité d'observateur au sein du Comité de développement institué cette année-là par la Banque mondiale et le FMI pour améliorer le transfert de ressources destinées au financement du développement. Dès 1977, la Suisse sera invitée également comme observateur aux assemblées générales des deux institutions.

En 1976, le peuple suisse refuse un nouveau crédit de 200 millions de francs à l'IDA au titre de la quatrième reconstitution de ses ressources, suite à un référendum lancé par le parti républicain de James Schwarzenbach ${ }^{5}$. Ce parti voulait marquer sa réticence vis-à-vis de la coopération au développement et plus particulièrement des institutions multilatérales. Dans la campagne précédant la votation populaire, d'autres voix critiques provenant d'une toute autre partie de l'échiquier politique se firent entendre: des organisations tiers-mondistes appuyèrent la campagne pour le 'non' à l'IDA pour faire connaître leur opposition à la politique de la Banque mondiale dans les pays en développement. Quelques jours avant le vote du 13 juin, un article paru dans Le Monde résume très bien la situation " Des gens vont voter 'non' parce qu'ils se veulent solidaires des

5. Le 13 juin 1976 l'arrêté fédéral proposant ce crédit est refusé par $56 \%$ des votants. Notons que si la contribution à l'IDA avait été faite sous la forme d'un don, il n'aurait pas pu donner lieu au lancement d'un référendum. En effet ce dernier n'était possible à l'époque que parce que l'accord avec I'IDA était un traité international d'une durée supérieure à 15 ans (en raison de la durée du prêt) soumis au référendum facultatif. 
plus pauvres du tiers-monde. D'autres vont voter 'oui' parce qu'ils espèrent des commandes pour notre industrie. Intérêt, xénophobie, fausse ou vraie solidarité sont inextricablement mêlés. II faudra se garder d'interprétations abusives le 13 juin au soir. On ne saura pas ce que le peuple suisse pense du tiers monde. ${ }^{m 6}$ Nous verrons plus loin quelles furent les conséquences de ce vote; disons d'ores et déjà qu'il ne facilita pas les relations avec la Banque.

En 1982, le Conseil fédéral prend la décision de principe de demander l'adhésion de la Suisse aux institutions de Bretton Woods sans se prononcer sur la date ni sur les conditions de cette demande d'adhésion: peu après, il sera décidé de donner la priorité à la demande d'adhésion à l'ONU.

En 1988, le réseau des relations avec le groupe de la Banque mondiale s'intensifie encore avec l'adhésion de la Suisse à l'AMGI et en 1990 avec la conclusion d'un accord avec la SFI.

\section{Les relations avec le groupe de la Banque mondiale}

\subsection{Le marché suisse des capitaux ouvert à la BIRD}

Le franc suisse occupe une place particulière dans les finances de la Banque mondiale, ce qui reflète l'importance du marché suisse des capitaux, les conditions relativement favorables d'emprunts offertes à des débiteurs de première qualité et le rôle international de la monnaie suisse

En 1948, lorsque la Banque emprunte pour la première fois dans une monnaie autre que le dollar américain, c'est en francs suisses qu'elle le fait. En 1951, la Suisse conclut avec la Banque mondiale un "Accord sur le statut juridique de la Banque internationale pour la reconstruction et le développement" destiné notamment à faciliter les émissions d'emprunts sur le marché suisse des capitaux. Aux termes de cet accord, la BIRD peut effectuer en Suisse toutes les opérations qui relèvent de son mandat - principalement lancement d'emprunts sur le marché des capitaux - après avoir obtenu l'accord de la Banque nationale suisse. Le point le plus important de cet accord est sans doute l'exonération de l'impôt anticipé sur le revenu des capitaux placés en Suisse et un taux préférentiel pour le droit de négociation (droit de timbre).

Au fil des ans, les emprunts de la Banque mondiale sur le marché suisse des capitaux se sont développés et dans les années 1980 la Banque devint le principal émetteur d'emprunts par obligations sur le marché suisse, le franc suisse étant une des principales monnaies dans lesquelles les emprunts de la Banque mondiale sont libellés. Le tableau I ci-dessous indique les montants empruntés, le pourcentage des emprunts en francs suisses par rapport au total des emprunts et le rang qu'occupe le franc suisse par rapport à d'autres monnaies.

6. Le Monde, 10 juin 1976. 


\section{Tableau I}

Importance du franc suisse dans les emprunts de la BIRD 1986-1990?

(en millions de dollars EU)

\begin{tabular}{|c|c|c|c|c|c|c|}
\hline \multirow[b]{2}{*}{ Période } & \multicolumn{3}{|c|}{ Avant swaps } & \multicolumn{2}{|c|}{ Après swaps } & \multirow[b]{2}{*}{ Rang } \\
\hline & Montant & $\%$ & Rang & Montant & $\%$ & \\
\hline 1986 & $1 ' 871,4$ & 18 & 2 & $3 ’ 095,8$ & 29 & 1 \\
\hline 1987 & $1 ' 494,7$ & 16 & 3 & $2 ' 286,1$ & 24 & 1 \\
\hline 1988 & $1 ' 117,0$ & 10 & 4 & 2055,1 & 19 & 3 \\
\hline 1989 & 586,5 & 6 & 4 & 966,4 & 10 & 3 \\
\hline 1990 & 745,0 & $\ldots$ & $\cdots$ & $\ldots$ & $\ldots$ & $\ldots$ \\
\hline \multicolumn{7}{|c|}{... données non disponibles pour 1990} \\
\hline
\end{tabular}

La Banque effectue ses opérations dans les diverses monnaies de ses membres, en DTS, ECU, en francs suisses. Pour équilibrer ses emprunts et ses prêts en diverses monnaies et pour toujours disposer au moment voulu des devises nécessaires au remboursement de ses emprunts, la BIRD conclut "des swaps de monnaies dans le cadre desquels le produit d'un emprunt en une monnaie donnée est converti en une autre monnaie; afin de recouvrer le montant de l'emprunt dans la monnaie d'origine, elle conclut simultanément un accord de change à terme qui comporte un échéancier selon lequel les deux monnaies concernées seront échangées. ${ }^{n 8}$ Le franc suisse joue également un rôle important dans ces transactions ainsi qu'en témoigne le tableau I. Au-delà des variations annuelles reflétées dans ce tableau on relève que le franc suisse est, avec le dollar des Etats-Unis, le yen japonais et le mark allemand, une des quatre principales monnaies.

Notons encore dans ce chapitre que, depuis 1988, la BIRD a lancé en Suisse un programme d'emprunt à court terme de "droits de paiement émis en continu". A la fin de l'exercice 1989, 62 millions de francs suisses avaient ainsi été empruntés. Ce programme "donne à la BIRD une flexibilité supplémentaire

7. Dans ce tableau comme dans les suivants, les années se réfèrent aux périodes couvertes par les exercices financiers de la Banque mondiale qui vont du 1 er juillet au 30 juin.

8. Banque mondiale, Rapport annuel 1989, p. 212 
dans la gestion de ses opérations de financement en francs suisses et répond à la demande des investisseurs pour de tels placements à court terme ${ }^{\text {mo }}$.

Que la BIRD ait fait appel dans une aussi large mesure au marché suisse des capitaux s'explique bien sûr en premier lieu par le niveau des taux d'intérêts qui, les rapports de la Banque l'attestent, ont été de façon suivie les plus bas en comparaison internationale jusqu'à la fin des années 1980. On peut aussi citer le coût réduit des frais d'émission et les autres avantages fiscaux consentis à la BIRD en 1951.

Le tableau I indique une tendance à la baisse de l'importance absolue et relative pour la Banque du marché suisse des capitaux. On peut dans une certaine mesure expliquer ce phénomène par une certaine saturation du marché des obligations de la Banque mondiale qui pourrait avoir pour effet d'accroître le coût des emprunts pour la Banque. Cette tendance est corroborée par l'évolution de la position créancière de la Suisse vis-à-vis de la BIRD pour ce qui est des emprunts à moyen et long terme, ainsi qu'en témoignent les données cidessous:

$\begin{array}{lcr}\begin{array}{l}\text { Année } \\ \text { situation } \\ \text { au 30 juin }\end{array} & \begin{array}{c}\text { Principal non amorti } \\ \text { en francs suisses } \\ \text { (exprimé en millions } \\ \text { de dollars) }\end{array} & \begin{array}{r}\text { Part du principal } \\ \text { en francs su } \\ \text { par rapport au }\end{array} \\ 1986 & 11^{\prime} 285 & \\ 1987 & 1^{\prime} 015 & 17,3 \% \\ 1988 & 1^{\prime} 590 & 17,4 \% \\ 1989 & 8^{\prime} 479 & 14,5 \% \\ 1990 & 8^{\prime} 051 & 11,3 \% \\ & & 9,9 \%\end{array}$

Source: Rapports annuels de la Banque mondiale de 1986 à 1990

\subsection{La promotion des investissements privés}

La loi suisse inclut dans les mesures de coopération au développement celles qui encouragent l'engagement de ressources du secteur privé, tels que les investissements qui favorisent le développement. Elle concorde ainsi avec les objectifs du groupe de la Banque mondiale qui a mis sur pied au fil des ans divers instruments de promotion de l'investissement privé dans les pays du Tiers monde.

9. Banque mondiale, Rapport annuel 1989, p. 68 


\subsubsection{La Sulsse et la Soclété financière Internationale $(\mathrm{SFI})^{10}$}

Depuis 1962, des banques suisses participent aux projets de la SFI en tant que co-financeurs et on estimait en 1987 que la Suisse est engagée dans environ 60 projets ${ }^{11}$. Sur le plan officiel, ont été accordées depuis 1986 des contributions à fonds perdus à cette institution pour un montant total de 1,8 million de dollars ${ }^{12}$. C'est ainsi que la Suisse a soutenu:

- l'Africa Project Development Facility qui aide de petites et moyennes entreprises à préparer des projets d'investissement, de co-entreprises et de transferts de technologie;

- le Foreign Investment Advisory Service qui conseille les pays en développement en vue d'attirer les investissements directs étrangers;

- I'African Management Services Company qui vise à améliorer la gestion des entreprises.

Jusqu'en 1984, la SFI empruntait directement ses ressources auprès de la BIRD. Son recours aux marchés des capitaux pour se procurer une partie de ses moyens financiers l'incita à s'intéresser au marché suisse des capitaux et à chercher à bénéficier des mêmes avantages que ceux dont jouit la BIRD en vertu de l'accord de 1951 (cf supra). C'est ainsi qu'un projet d'accord est soumis à la ratification des Chambres fédérales par un Message du 21 février 1990. II correspond à celui de 1951, en particulier quant aux avantages fiscaux octroyés aux emprunts de la SFI qui jouiront d'un taux préférentiel pour le droit de négociation et d'une exonération de l'impôt anticipé.

\subsubsection{La participation de la Suisse à l'AMGI}

En 1985 la Banque a mis sur pied une agence multilatérale de garantie des risques de l'investissement - AMGI (MIGA en anglais) dont le but est de protéger contre les risques politiques, les capitaux étrangers investis dans les pays en

10. La SFI, fondée en 1956, fait partie du groupe de la Banque mondiale; elle est ouverte aux Etats membres de la Banque. Son objectif spécifique est de promouvoir le développement d'entreprises privées dans les pays en développement en participant directement à leur capital social ou en leur octroyant des crédits. Elle ne finance qu'une partie du coût d'un projet (jusqu'à $25 \%$ ). Actuellement, la SFI emprunte directement sur le marché des capitaux la moitié environ des crédits dont elle a besoin (environ 250 millions de dollars par an. Source: Message du 21 février 1990, p1659.

11. Voir B. Hürni (1980) et K.Vökl (1987)

12. Toutes les données figurant ci-dessous sur la SFI sont tirées du Message du 21 février 1990 pp.1658 à 1663. 
développement. L'AMGI couvre les risques de transfert ainsi que ceux qui sont liés aux expropriations, aux violations de contrats et aux situations de guerre et de guerre civile.

La Suisse a d'emblée décidé de participer à cette nouvelle filiale de la Banque mondiale qui a commencé ses activités en juin 1988. Elle est le seul pays non membre de la Banque à l'avoir fait. Le Conseil fédéral avait considéré dans son Message que les risques politiques freinaient effectivement les investissements privés dans les pays en voie de développement et que les instruments bilatéraux sont insuffisants. La contribution de la Suisse, membre fondateur est de 15,5 millions de dollars, soit 1,5\% du capital de l'AMGI. $10 \%$ de ce montant a été versé en 1988; le solde est un montant garanti. ${ }^{13}$

\subsubsection{La participation de la Suisse au CIRDI}

Le Centre international pour le règlement des différends relatifs aux investissements a été créé en 1965 à l'initiative de la Banque mondiale. La Suisse en est le seul des 91 membres qui ne soit pas membre de la Banque mondiale. Le CIRDI, en offrant un cadre de conciliation et d'arbitrage, veut contribuer à améliorer le climat d'investissement et encourager les flux privés d'investissement. II poursuit en cela les mêmes objectifs que l'AMGl et les raisons qu'eut la Suisse d'y adhérer sont fondamentalement les mêmes que celles évoquées au paragraphe précédent.

\subsection{Les relations avec I'IDA}

\subsubsection{Vue d'ensemble: les trois phases des relations}

Depuis sa création en 1960, I'IDA s'est essentiellement procuré ses ressources auprès des pays membres qui ont constitué au départ un fonds initial de ressources et qui ont par la suite contribué à neuf reprises à la reconstitution de ses ressources (cf. le tableau II ci-dessous). La reconstitution des ressources de I'IDA ne s'est pas toujours réalisée sans peine. De longues négociations, dues en particulier aux réticences des Etats-Unis, retardèrent à plusieurs reprises les calendriers prévus et provoquèrent un ralentissement des opérations de l'IDA. De même, les réserves américaines empêchèrent parfois la reconstitution d'atteindre un niveau jugé convenable, au regard notamment du volume des reconstitutions précédentes et des besoins accrus de l'IDA provoqués par l'entrée de nouveaux membres, en particulier de la Chine.

13. L'Annualre Suisse-Tlers Monde 1987/88, no 7, pp.73-76, rapporte les raisons qui ont amené la Suisse à adhérer ainsi que les réactions critiques suscitées par la proposition d'adhésion. 
L'histoire des relations de la Suisse avec I'IDA peut être divisée en trois phases:

A. De 1969 à 1976: participation de la Suisse à la deuxième et troisième reconstitution des ressources de l'IDA par des prêts accordés à des conditions de faveur (notons que les contributions de tous les membres de l'IDA sont à fonds perdus); cette phase se termine le 13 juin 1976 avec le refus populaire d'une participation de Frs 200 millions à la quatrième reconstitution.

B. De 1976 à 1984, la Suisse gère tant bien que mal le choc représenté par ce refus. La Suisse ne participera donc pas formellement à cette reconstitution, pas plus qu'aux cinquième et sixième mais opèrera un rapprochement de I'IDA par une série de mesures ponctuelles:

- pendant la période IDA-4 et IDA-5, les seules participations de la Suisse aux activités de l'IDA furent le cofinancement de trois projets (un au Bangladesh et deux en Afrique sahélienne) pour un montant de 46, 2 millions de francs;

- au début de la période de IDA-6, selon une décision parlementaire d'octobre 1980, il sera procédé à une transformation des prêts accordés à I'IDA en 1967 et 1972 en dons. Cette opération - qui s'imposait pour donner au groupe de la Banque mondiale un signe concret de l'intérêt des autorités suisses à poursuivre la collaboration avec lui - représentait un don dont la valeur actualisée était d'environ 28 millions de francs; en second lieu, pendant cette même période une série de cofinancements ponctuels de projets pour un montant de 84,25 millions de francs fut entrepris.

C. A partir de 1985 (début de la $7 e$ reconstitution), une nouvelle phase de collaboration s'ouvre avec la conclusion d'accords-cadre portant sur la participation de la Suisse aux reconstitutions successives des ressources de l'IDA. Dans ces accords qui portent sur IDA-7, IDA-8 et IDA-9, le Conseil fédéral s'engage quant au montant des ressources financières que la Suisse entend mettre à disposition de I'IDA pour la réalisation de cofinancements durant la période prévue pour la reconstitution. C'est sur cette dernière phase que portera notre analyse en nous concentrant sur les engagements pris au titre de IDA-7 et IDA-8.

Dans le cadre de cette troisième phase, il convient également de mentionner la participation de la Suisse à des opérations spéciales de l'IDA en faveur de l'Afrique:

- Le Fonds spécial d'aide à l'Afrique subsaharienne (FSA) $)^{14}$ : par un accord du 11 septembre 1985, la Suisse s'engageait à fournir à l'IDA une contribution

14. Ce Fonds avait pour but de fournir une aide à décaissement rapide aux pays qui entreprennent des réformes structurelles jugées appropriées par la Banque mondiale. Cette aide fournie par 18 pays et la Banque mondiale est venue s'ajouter aux ressources mises à disposition dans le cadre d'IDA-7. 
de 80,4 millions de francs pour réaliser des cofinancements durant la période allant de juillet 1985 à juin 1988.

\section{Tableau II}

\section{Reconstitution des ressources de I'IDA 1961-1990 et contributions de la Sulsse}

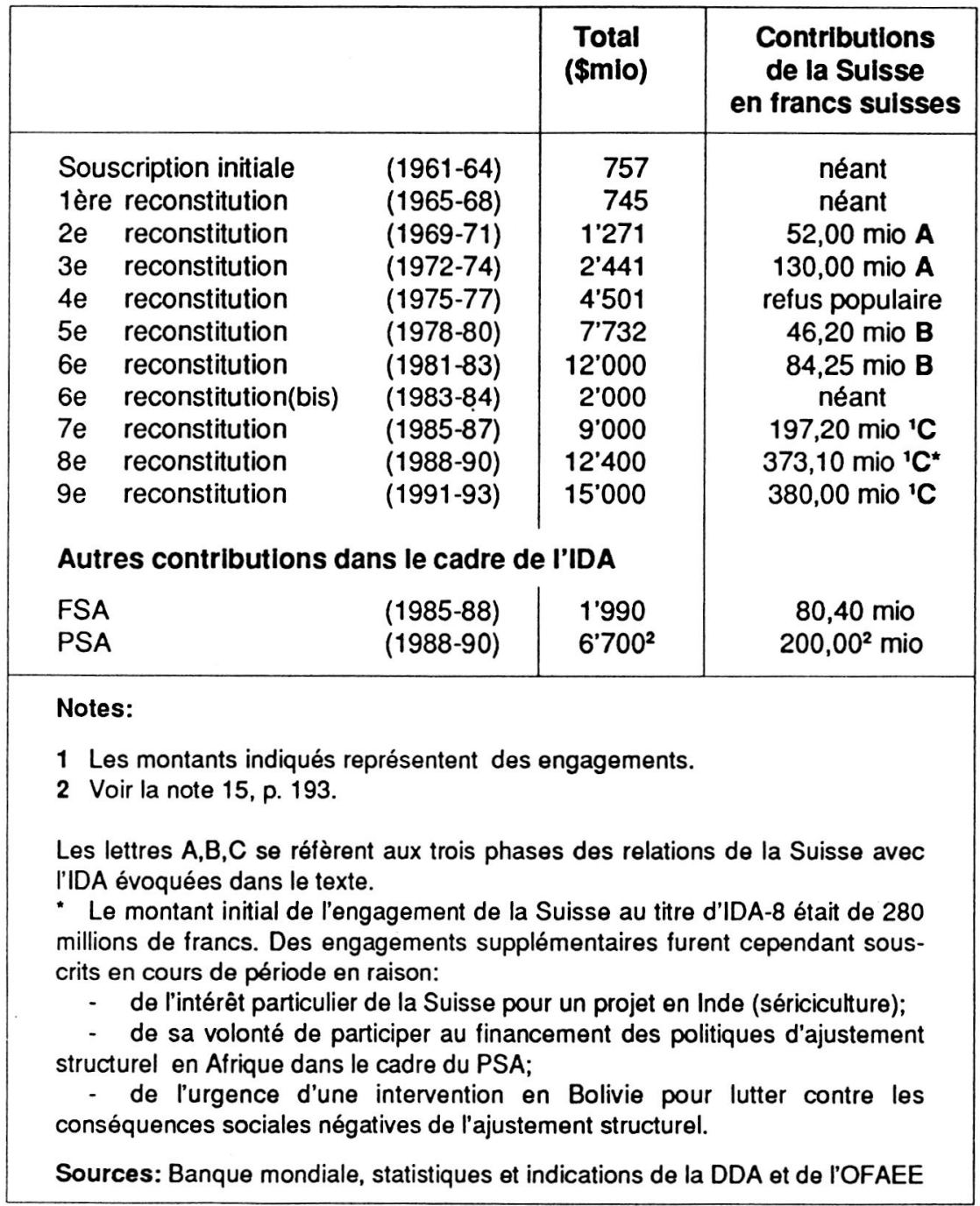


- Le Programme spécial d'assistance pour l'Afrique (PSA) ${ }^{15}$ : la Suisse décida de participer à ce programme pour un montant de 200 millions de francs, mais seulement la moitié environ de cet engagement représentait des ressources additionnelles. L'autre moitié étant incluse dans l'engagement déjà pris au titre d'IDA-8.

La collaboration de la Suisse avec l'IDA comprend également la gestion d'un SwIss Consultants Trust Fund. Depuis 1985, la Suisse (par le biais de la DDA) alimente ce fonds destiné à financer des missions de consultants et de bureaux d'études suisses dans le cadre de projets de l'IDA. Une petite partie de ces ressources peut être engagée pour financer des consultants de pays en développement.

Le "STABEX sulsse": Dans le cadre des mesures de politique économique et commerciale, l'OFAEE a lancé dès 1988 un programme destiné à compenser des pertes de recettes d'exportation des six pays africains classés dans la catégorie des PMA ayant enregistré en 1986 et 1987 les pertes les plus importantes dans leurs exportations vers la Suisse. Les montants ont été calculés selon les règles du STABEX de la Communauté européenne. Dans quatre des six cas, les versements compensatoires ont été effectués sous forme de cofinancements dans le cadre de programmes et de projets d'ajustements sectoriels de I'IDA. Ces contributions concernant quatre pays pour un montant de 13,5 millions de francs ont été comptabilisées dans la contribution de la Suisse au titre de IDA-8.

La part de la Suisse dans la $9 e$ reconstitution des ressources de I'IDA s'élève à $1,58 \%$ du total. Cette contribution est inférieure à ce que versent d'autres pays industrialisés dont le PNB est du même ordre de grandeur que celui de la Suisse.

15. A la fin de 1987, la Banque mondiale invitait les pays industriels à prendre une nouvelle initiative en faveur des pays africains écrasés par le surendettement. II s'agissait de mettre sur pied un programme prévoyant des financements accrus, fortement concessionnels et à décaissement rapide ainsi qu'un allégement de la dette pour accroître la capacité d'importation des pays en voie d'ajustement. (Rapport de la Banque mondiale 1989, p. 43). II s'agissait d' inviter les pays fournisseurs d'aide à agir sur plusieurs fronts et notamment par:

- un accroissement des crédits à l'ajustement financés au titre d'IDA-8;

- un accroissement des cofinancements pour l'ajustement;

- un financement accru du FMI au moyen de la facilité d'ajustement structurel renforcée;

- un allègement concessionnel de la dette.

Pour une partie de ces mesures (environ $50 \%$ ), il ne s'agisssait pas - contrairement au FSA - de fournir des ressources additionnelles mais d'affecter des ressources déjà engagées à la réalisation de ce programme. 
Pays

Suisse

Belgique

Pays-Bas

Suède
PNB (1988)

$\$$ milliards

184

153

228

159
Part dans IDA-9
$1,58 \%$
$1,55 \%$
$3,00 \%$
$2,62 \%$

\subsubsection{Les cofinancements avec I'IDA}

Dans le présent contexte on peut définir les cofinancements comme une contribution à une institution multilatérale destinée à participer au financement d'un projet ou d'un programme spécifique de cette institution. Le cofinancement se distingue des contributions générales au programme d'une institution multilatérale: il permet en effet au financeur de déterminer de façon précise le destinataire de sa contribution ainsi que la nature des prestations à laquelle elle sera affectée. Dans le cadre de la coopération au développement, les cofinancements sont classés dans l'aide bilatérale alors que les contributions générales sont enregistrées au titre de l'aide multilatérale. En général, dans le cadre de I'IDA, les cofinancements permettent aux membres de cette institution d'apporter volontairement, au-delà de leur contribution générale au titre de la reconstitution des ressources, un appui spécifique à un pays et/ou à un secteur qui pour des raisons diverses leur semble prioritaire. De même le cofinancement a-t-il été largement utilisé pour financer le programme spécial d'assistance à l'Afrique (PSA).

Dans le cas de la Suisse, la pratique des cofinancements avec l'IDA est née d'une nécessité dès le moment où le peuple suisse s'était prononcé - pour des raisons diverses - contre une participation de la Suisse à la quatrième reconstitution des ressources de l'IDA. En effet, le résultat du vote ne pouvait être pour le moins interprété que comme un désaveu à l'égard de la coopération multilatérale en général et de la Banque mondiale en particulier à l'égard desquelles de vives critiques avaient été émises lors de la campagne précédant le vote. On ne pouvait donc ignorer le résultat de ce vote et continuer à participer aux reconstitutions des ressources de l'IDA. Le Conseil fédéral en tirera la conclusion que la part de l'aide multilatérale, peu populaire, ne devait pas être augmentée et, de fait, elle sera maintenue au même niveau relatif, oscillant autour de $25 \%$ de l'APD totale au cours des années $1980^{16}$. En second lieu, il ne sera plus

16. La diminution de la part de l'aide multilatérale qui apparaît dans les statistiques de l'aide suisse à partir de 1980 s'explique essentiellement par un changement dans la présentation des statistiques. Dès cette date, les contributions à des organisations internationales pour le financement de projets et programmes spécifiques ne figurent plus dans l'aide multilatérale mais dans l'aide bilatérale. 
Tableau III

Les cofinancements de la Suisse dans le cadre de I'IDA 1975-1990

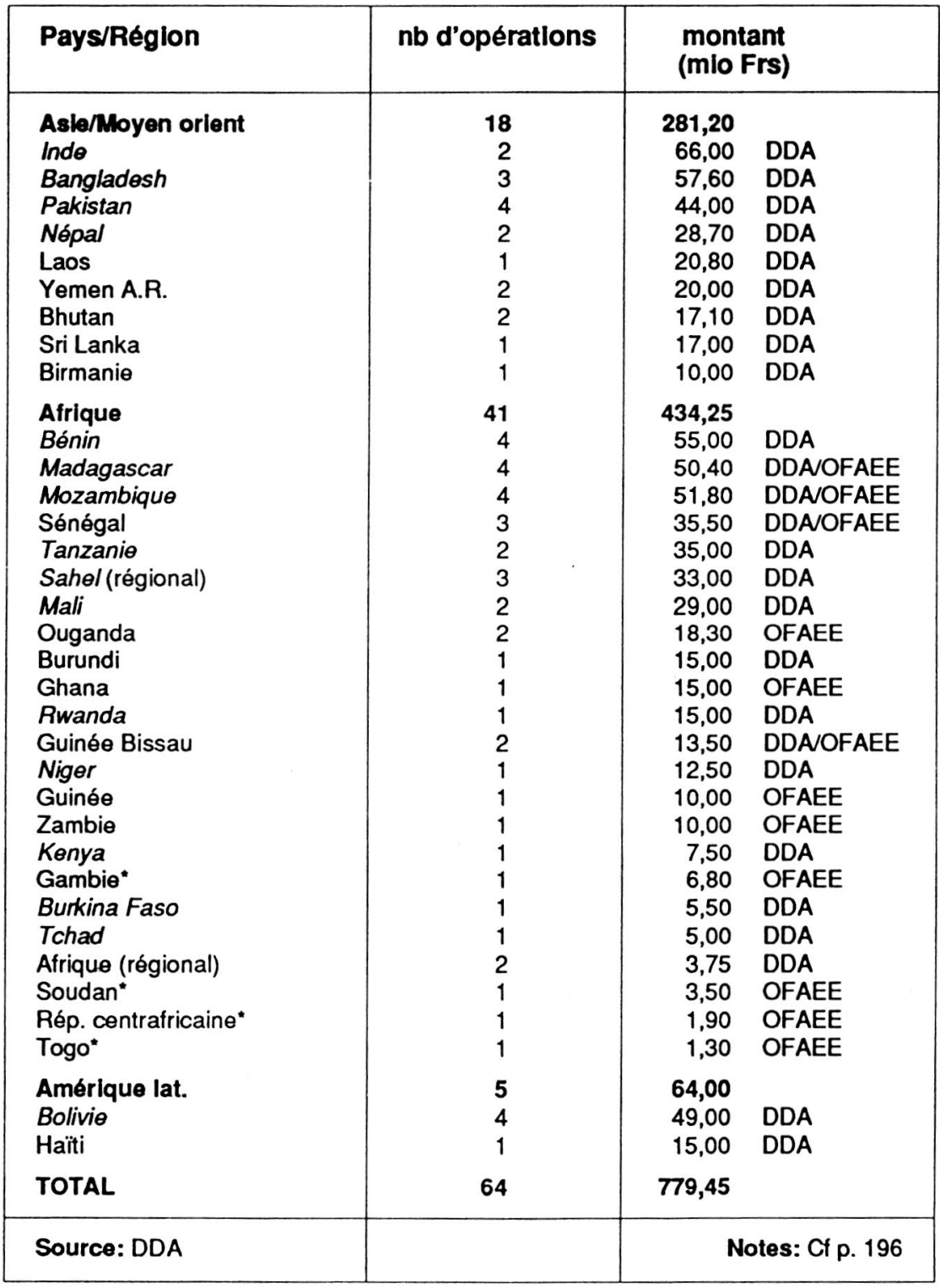




\section{Notes}

- Opérations effectuées dans le cadre du "STABEX suisse"

- Les pays en caractères italiques représentent les pays de concentration de la coopération suisse au développement

- Les montants ci-dessus représentent les engagements pris par la Suisse depuis 1975. Ils incluent l'engagements pris au titre du Fonds spécial pour l'Afrique subsaharienne (80,4 millions de francs de 1985 à 1988). Ils n'incluent pas les engagements pris au titre de IDA-9 (1990-1993).

\section{Tableau IV}

Versements au titre de cofinancements avec I'IDA (millions de francs)

\begin{tabular}{|l|r|}
\hline Années antérieures & 45,05 \\
1984 & 21,27 \\
1985 & 48,50 \\
1986 & 71,25 \\
1987 & 84,30 \\
1988 & 60,90 \\
1989 & 84,90 \\
Total cumulé & 416,17 \\
\hline
\end{tabular}

question de participer par de nouvelles contributions générales aux reconstitutions suivantes des ressources de l'IDA. Le vote de 1976 n'empêchera cependant pas la Suisse de devenir membre de plein droit des grandes banques régionales de développement et de leurs fonds spéciaux en faveur des pays à bas revenus.

II était cependant tout aussi difficile d'envisager de rester totalement à l'écart des activités de la Banque mondiale. A la Banque mondiale et dans quelques capitales de pays industrialisés on imaginait mal que les entreprises suisses continuent de pouvoir livrer des biens et des services financés par des fonds de I'IDA dès lors que la Suisse ne participait plus à son financement. A plusieurs reprises, le président de la Banque mondiale viendra en Suisse répéter ce message et les milieux de la coopération internationale au développement saisissaient de plus en plus mal que la Suisse riche et stable profite des avantages de la participation au club sans acquitter de cotisations! Les limites du Sonderfall étaient atteintes.

Un premier geste du gouvernement suisse sera de participer par des décisions ponctuelles au cofinancement de certains projets de l'IDA. Puis, en 1981, les deux prêts accordés à l'IDA furent convertis en dons. 
Les contributions de la Suisse sont faites sous forme de dons et sont déliées. Les biens et services financés par la contribution suisse sont acquis conformément aux règles de la Banque mondiale.

Les cofinancements réalisés dans les pays ou régions de concentration de la cooperation suisse représentent 479 millions de francs, soit $61,5 \%$ du total.

\section{Répartition des cofinancements entre la DDA et l'OFAEE}

Les cofinancements de la Suisse sont financés soit par le crédit de programme de coopération technique et financière géré par la DDA ou par le crédit de programme pour les mesures de politique économique et financière géré par I'OFAEE. Au cours des trois dernières reconstitutions, la répartition des financements a été la suivante:
IDA-7
DDA: 170,0 mio
OFAEE: $\quad 25,5$ mio
IDA-8
DDA: 296,2 mio
OFAEE: $\quad 76,9$ mio
IDA-9
DDA: 260,0 mio
OFAEE: 120,0 mio

\section{La pratique des cofinancements}

Les modalités générales de la coopération de la Suisse avec l'IDA en matière de cofinancements sont réglées par un accord (le dernier en date portant sur IDA-9 a été signé le 9 mai 1990). Chaque cofinancement est construit sur le plan juridique sur trois accords bilatéraux:

- entre l'IDA et le pays bénéficiaire;

- entre la Suisse et le pays bénéficiaire;

- entre la Suisse et l'IDA.

\section{Le choix des projets et des programmes cofinancés}

Les projets et programmes au financement desquels la Suisse participe sont déterminés d'un commun accord. En pratique l'initiative de proposer un cofinancement peut venir aussi bien de la Suisse que de I'IDA. La Suisse peut participer aux missions de préparation et d'évaluation ex ante des projets et programmes ainsi qu'aux négociations entre l'IDA et le bénéficiaire. La décision de cofinancement est finalement prise avec l'accord du pays bénéficiaire.

Les critères de choix des cofinancements varient selon qu'il s'agit de la DDA ou de l'OFAEE.

Dans le cas de la DDA on cherchera en premier lieu une articulation avec le programme de coopération technique et financière de la Suisse. C'est dire que l'on choisira de façon prioritaire des pays de concentration. Ce choix permet non 
seulement d'accroître l'impact de la cooperation suisse dans un pays, mais encore de pouvoir mettre à profit les connaissances que l'on a du pays en question et de la présence permanente sur place de collaborateurs de la DDA. En outre, le choix du projet ou de la composante du projet à financer devrait aussi être fait en fonction de l'expérience opérationnelle de la DDA et de sa capacité à faire appel à des experts dans ce domaine pour la conseiller. Ce critère de choix prend tout son sens dans la mesure où la DDA souhaite participer activement à la préparation des projets à cofinancer. Les avantages des cofinancements par rapport à la coopération purement bilatérale peuvent être significatifs pour le petit fournisseur d'aide qu'est la Suisse: ils permettent de participer à de grands projets qui ne pourraient jamais être financés uniquement par des ressources d'origine suisse et de prendre part à des discussions de politique sectorielle auxquelles un petit donneur bilatéral ne serait pas convié.

Dans le cadre d'IDA-7 et d'IDA-8 seuls 7 cofinancements de la DDA sur 31 eurent lieu dans des pays autres que les pays de concentration pour un montant de 79,9 millions de francs sur un total de $\mathbf{4 6 6 , 2}$ millions. L'avantage des cofinancements est ici de pouvoir être présent dans des pays où il n'y a par ailleurs pas de programme bilatéral.

Pour l'OFAEE les critères sont différents car les cofinancements se sont situés dans le cadre de programmes d'ajustement structurel auxquels I'IDA participe. L'urgence et l'importance des besoins en devises jouent un rôle dans le choix des pays bénéficiaires ainsi que la qualité des programmes de réformes, leurs perspectives de succès, c'est-à-dire le potentiel de récupération économique du pays, en particulier dans le domaine de ses relations économiques extérieures. II sera en outre aussi tenu compte des relations économiques et politiques de la Suisse avec les pays bénéficiaires. Dans plusieurs cas une opération de cofinancement a préparé le terrain pour une deuxième opération dans le même pays, conduite cette fois de façon purement bilatérale.

\section{Le sulvi et l'évaluation des projets et programmes cofinancés}

Les projets et programmes cofinancés sont réalisés sous la responsabilité de I'IDA. La Suisse est invitée à participer aux missions de suivi ou d'évaluation des projets et programmes cofinancés pour autant que le pays bénéficiaire soit d'accord. Dans l'accord du 9 mai 1990, il est prévu pour la première fois que la Suisse puisse conduire de façon indépendante une évaluation ex post d'une opération de cofinancement. L'IDA peut y être associée si elle le désire. La Banque mondiale dispose d'un système complexe de suivi et d'évaluation de ses projets. La Suisse a donc de multiples occasions de participer à ce processus, les limites de cette participation étant la disponibilité de personnel suffisamment qualifié et expérimenté pour pouvoir jouer un rôle dans ce processus. Dans la pratique et vu le nombre relativement important d'opérations de cofinancement, l'administration fédérale est amenée à concentrer ses efforts sur certaines opérations qu'elle juge particulièrement importantes et/ou instructives, ou dans lesquelles la Suisse dispose d'une expertise certaine et reconnue. 


\subsection{La fourniture par des entreprises suisses de blens et de services finances par la Banque mondlale}

En raison de l'étroite collaboration de la Suisse avec le groupe de la Banque mondiale dans le domaine financier, les entreprises suisses peuvent participer aux appels d'offres internationaux pour la fourniture de biens et de services financés par la Banque mondiale (BIRD et IDA). La Suisse est ainsi le seul Etat non-membre à bénéficier de cette possibilité qui est mentionnée explicitement dans les directives concernant la passation des marchés financés par les prêts de la BIRD et les crédits de l'IDA.

\section{Tableau V}

Décaissements de la BIRD et de l'IDA au titre de marchés passés avec des entreprises suisses

(montant en millions de dollars des Etats-Unis et part des entreprises suisses dans le total des décaissements)

\begin{tabular}{|c|c|c|c|c|c|}
\hline & \multicolumn{2}{|c|}{ BIRD } & \multicolumn{2}{|c|}{ IDA } & Total \\
\hline & montant & $\%$ & monitant & $\%$ & montan \\
\hline 1986 & 182,0 & $4,0 \%$ & 68,4 & $3,2 \%$ & 250,4 \\
\hline 1987 & 285,6 & $4,0 \%$ & 69,6 & $3,1 \%$ & 355,2 \\
\hline 1988 & 288,7 & $4,2 \%$ & 68,1 & $2,7 \%$ & 356,8 \\
\hline 1989 & 317,0 & $4,7 \%$ & 69,0 & $2,8 \%$ & 386,0 \\
\hline 1990 & 323,0 & $3,5 \%$ & 36,0 & $1,4 \%$ & 359,0 \\
\hline $\begin{array}{l}\text { Total cum. } \\
\text { au } 3.6 .90\end{array}$ & $3^{\prime} 250,0$ & $4,0 \%$ & 698,0 & $2,7 \%$ & $3 ' 948,0$ \\
\hline
\end{tabular}

Pour l'exercice 1990 (juillet 1989-juin 1990), la part du marché obtenue par les entreprises suisses se présentait ainsi pour différentes catégories de prestations de biens et de services (BIRD et IDA confondues):

- équipements

- travaux de génie civil

- consultants

- autres biens

- total
$\$ 182$ mio

$\$ 36 \mathrm{mio}$

$\$ 17 \mathrm{mio}$

$\$ 125$ mio

$\$ 359$ mio
$4,2 \%$

$6,9 \%$

$2,9 \%$

$2,9 \%$

$3,0 \%$ 
On notera la part plus faible des consultants dans les données ci-dessus. Les entreprises suisses concernées sont-elles moins compétitives ou moins intéressées? Toujours est-il que la Confédération, comme d'autres pays européens, a établi auprès de l'IDA un fonds spécial de $\mathrm{Fr}$ 2,2 millions destiné à financer les prestations de firmes suisses de consultants.

Le tableau $\mathrm{V}$ ci-dessus indique les montants absolus et limportance relative des décaissements de la BIRD et de l'IDA au titre de marchés passés avec des entreprises suisses de 1986 à 1990 et le total cumulé au 30 juin 1990 depuis le début des opérations de la Banque. Ce qu'il n'indique pas, c'est la place privilégiée de la Suisse dans le tableau d'ensemble de ces décaissements: elle se trouve en effet au 7ème rang après les grandes puissances industrielles occidentales que sont les Etats-Unis, le Japon, l'Allemagne, la France et le Royaume-Uni et l'Italie. On notera que les entreprises suisses ont dans l'ensemble beaucoup plus bénéficié des achats financés par la Banque mondiale que celles de pays comparables quant à la structure et la dimension de leur économie (Suède, Pays-Bas, Belgique), et même que le Canada dont le PNB est environ 2,5 fois plus élevé que celui de la Suisse. II convient en outre de noter que le maintien de la position de la Suisse se situe dans un contexte où la part des pays en développement aux décaissements tend à augmenter, ayant passé pour la BIRD de $15,8 \%$ en 1986 à $23,5 \%$ en $1990 .{ }^{17}$

En mettant en regard les versements suisses à l'IDA dans le cadre de cofinancements et le montant des commandes aux entreprises suisses financées par I'IDA, on constate que ce dernier dépasse régulièrement et substantiellement les premiers. Ceci signifie que d'autres contributeurs à l'IDA financent une partie des livraisons des entreprises suisses. Dans le contexte économique international actuel cette situation risque d'être de plus contestée par des pays industrialisés qui en matière d'APD en font sensiblement plus que la Suisse. Cette situation indique aussi que les entreprises suisses sont concurrentielles sur le marché mondial; la possibilité d'acheter des biens et des services en Suisse est donc un avantage aussi pour les pays utilisateurs des ressources de l'IDA.

\subsection{Autres formes de cooperation avec la Banque mondlale}

Au fil des ans, la Suisse a développé une collaboration ponctuelle avec plusieurs programmes de la Banque mondiale: mentionnons pour mémoire:

17. En 1980, dans l'article qu'elle consacra à la question de l'adhésion de la Suisse à la Banque mondiale, Bettina Hürni évoquait cette question en notant que la Suisse avait relativement peu à craindre de la concurrence des pays en développement:

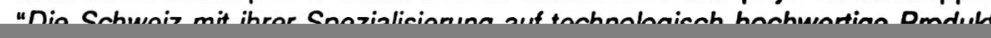


- le Programme eau/assainissement géré par la Banque mondiale et le PNUD;

- le Programme des transports au Sud du Sahara;

- le programme sur les dimensions sociales de l'ajustement (Banque mondiale/PNUD).

\section{Les enjeux de l'adhésion}

Nous l'avons dit au début de cet article, la question de la Banque mondiale n'est pas véritablement au centre du débat sur l'adhésion de la Suisse. II n'en reste pas moins qu'elle pèse fortement dans le débat en raison du poids de cette institution dans les relations Nord-Sud et de l'importance des relations qu'elle entretient avec la Suisse. Les questions qui se posent face à une proposition d'adhésion sont les suivantes:

- quel bilan peut-on établir de nos relations actuelles ?

- en quoi et comment une adhésion pourrait-elle modifier ce bilan ?

Au moment où nous rédigeons cet article, nous ne disposons pas des éléments qui font partie de la négociation en cours entre le Conseil fédéral et les institutions de Bretton Woods. Nous laisserons donc de cóté les aspects liés au montant de la souscription de la Suisse au capital social de la BIRD, des contributions à l'IDA et des droits de vote qui en résulteraient. Nous nous concentrerons sur deux autres aspects majeurs des relations de la Suisse avec la Banque mondiale:

- les relations financières et économiques

- les relations dans la formulation et la réalisation de la coopération au développement.

\subsection{Les enjeux économiques et financiers}

Les relations qui se sont instaurées avec la BIRD, pour ce qui est du financement de ses activités, sont importantes aussi bien pour la Banque que pour la Suisse. Cependant leur avenir dépend beaucoup plus de l'évolution des conditions sur le marché suisse des capitaux que de l'adhésion ou de la non adhésion de la Suisse. On peut penser que dans l'avenir, les conditions qui prévaudront sur ce marché tendront de plus en plus à s'aligner sur les conditions du marché international, en particulier en Europe, et que le róle relatif de la place financière suisse et du franc suisse dans les finances de la Banque mondiale tendra à diminuer. C'est ce que semble indiquer la tendance des cinq dernières années, même sị la Suisse restera très probablement un pays exportateur de capitaux. 
Sur le plan économique, les effets pour les entreprises suisses des achats de biens et de services financés par la Banque mondiale seront en principe peu touchés par une adhésion. Les facteurs déterminants sont la compétitivité de nos entreprises et la capacité de conserver cette possibilité qu'ont les entreprises suisses de participer aux appels d'offre internationaux dans le cadre de projets et programmes financés par la Banque mondiale. Le second élément ne pose aucun problème en cas d'adhésion. En cas de non adhésion ou de refus d'adhésion le maintien de cette situation spéciale dépendra de l'importance que conservera le marché suisse des capitaux pour la BIRD et du maintien à un niveau jugé acceptable par les membres de la Banque mondiale d'une contribution suisse aux reconstitutions des ressources de l'IDA.

\subsection{Les enjeux sur le plan de la coopération au développement}

L'examen de ces enjeux requiert une appréciation des convergences et des divergences qui peuvent exister entre la Banque mondiale et la Suisse sur le plan de la politique de coopération au développement. En effet, l'adhésion à une institution quelle qu'elle soit pose d'autant moins de problèmes à l'impétrant qu'il peut s'identifier avec ses objectifs et ses activités. La Suisse participe au financement des activités de l'IDA parce qu'elle reconnaît le rôle important joué par la Banque mondiale dans la coordination de l'aide, la nécessité de l'aide financière multilatérale pour mettre en oeuvre des projets et programmes de grande envergure. De plus, l'attention exclusive que porte l'IDA aux pays à faibles revenus est conforme aux objectifs de la loi sur la coopération internationale. On peut sans doute affirmer qu'il n'y a pas de divergences de fond entre les autorités suisses et l'IDA. Dans les opérations de cofinancement, la Suisse cherche à privilégier par son apport certaines composantes d'un programme ou d'un projet. Les aspects sociaux et écologiques sont pour elle particulièrement importants. Elle veut en outre mettre à profit son expérience particulière dans certains secteurs ou pays pour influencer la formulation d'un projet ou d'un programme dans un sens qui lui paraît mieux correspondre aux priorités de la politique suisse de coopération au développement.

Dans le contexte des cofinancements, on peut penser que l'impact de la Suisse est en général limité et aléatoire, bien que la situation puisse varier considérablement d'un cas à l'autre. La capacité qu'a la Suisse d'influencer une opération de l'IDA dépend

- du pays et du secteur dans lequel le programme se déroule;

- de l'importance relative du financement suisse dans le financement d'ensemble du projet. A cet égard, la situation est variable: quelques exemples tirés de projets cofinancés dans le cadre de l'IDA-8 montrent que la part du financement suisse peut osciller entre plus de $60 \%$ dans des projets relativement restreints (dans le secteur de la santé au Bénin par exemple) et moins de $5 \%$ dans de gigantesques projets industriels (industrie électronique en 
Inde dont le coût total est de $\$ 450$ millions et la part de la Suisse $\$ 16,6$ millions);

- du stade de la préparation du projet ou programme à partir duquel la Suisse est associée au projet; une intervention précoce est recommandée;

- de la capacité de l'administration fédérale de mobiliser des experts pour participer à des missions de préparation de projets et programmes.

Dans sa réponse à l'interpellation Pitteloud, le Conseil fédéral montre clairement que les possibilités de participation de la Suisse à la réalisation de projets et programmes sont théoriquement très nombreuses, dans la pratique des limites sont fixées par le manque de personnel ainsi que par le poids relativement faible de la Suisse dans les financements. ${ }^{18}$

C'est sur le plan de la participation à la formulation de la politique des institutions de Bretton Woods que les enjeux de l'adhésion sont les plus importants. En effet, si la Suisse a réussi au fil des ans à négocier avec la Banque mondiale un statut tout à fait unique lui permettant d'être associée à la plupart des activités de l'institution et à bénéficier de certains droits réservés en principe aux seuls membres, il lui manque un attribut essentiel: le droit de participer de plein droit et de voter au sein des instances décisionnelles de la Banque mondiale. Jusqu'ici, la Suisse a joué au sein de l'institution un róle marginal par rapport à la place qu'elle occupe dans les relations économiques internationales. Sa participation en qualité d'observateur au Comité de développement et aux assemblées générales de la Banque, sa participation aux groupes consultatifs de divers pays auxquels elle est directement intéressée dans le cadre de sa politique de coopération au développement, les consultations, concertations et accords dans le cadre de cofinancements représentent certes des occasions de participer aux grands - et moins grands - débats de politique de développement, mais le poids décisionnel est le plus souvent absent et lorsqu'il peut s'exercer, ce n'est que de façon ponctuelle dans le cadre de projets ou programmes cofinancés.

Si la Suisse adhérait à la Banque mondiale, une conséquence importante serait sinon la disparition, du moins une très forte diminution des cofinancements avec I'IDA. En effet la Suisse serait alors tenue de fournir ses apports au titre des reconstitutions des ressources de l'IDA sous forme de contributions générales comme le font les autres membres. II est clair que la Suisse pourrait, en plus de cette contribution "ordinaire" mettre à disposition de certains projets et programmes de I'IDA des ressources supplémentaires sous la forme de cofinancements, mais cette pratique serait sans aucun doute beaucoup plus limitée qu'aujourd'hui. Que représenterait ce changement pour les divers acteurs concernés?

18. Réponse du Conseil fédéral à une interpellation Pitteloud sur les cofinancements IDA-DDA (88.331). 
Pour l'IDA, ce serait certainement un soulagement. En effet la préparation, la négociation et la gestion de cofinancements est plus lourde que les procédures ordinaires. Le jeu en vaut la chandelle si ces cofinancements apportent à l'IDA des ressources supplémentaires par rapport à la contribution des pays donateurs au titre de la reconstitution des ressources. Ce n'est pas le cas pour la Suisse qui ne contribue en tout cas pas plus que la part qui serait la sienne si elle était membre de l'institution.

Vus des pays bénéficiaires, les cofinancements peuvent être considérés favorablement s'ils apportent des ressources supplémentaires. L'intervention d'un tiers dans la négociation avec l'IDA peut compliquer le processus, en particulier si le cofinanceur ajoute sa conditionnalité à celle de l'IDA (conditionnalité croisée). On peut aussi cependant imaginer qu'un désaccord entre un cofinanceur et l'IDA renforce finalement le pouvoir de négociation du bénéficiaire. Mais ce n'est pas le cas de figure le plus fréquent ni forcément le plus souhaitable.

Du point de vue de la Suisse enfin, la question ne se pose guère dans la mesure où il est évident - en tout cas sur le plan théorique - que la volonté d'exercer une influence sur la Banque mondiale qui transparaît constamment dans le discours des autorités serait mieux servie par une adhésion que par le maintien du statu quo. ${ }^{19}$ Cependant le plein exercice des droits d'un membre de la Banque mondiale requiert que les ressources humaines et l'organisation de l'administration fédérale soient développées pour pouvoir suivre, non seulement quelques dizaines d'opérations de cofinancement mais l'ensemble de la politique et des opérations de ce géant qu'est la Banque mondiale. II s'agit là d'une tâche nouvelle importante qui doit être prise au sérieux pour que l'adhésion prenne véritablement son sens. Or, on a vu plus haut que le manque de personnel qualifié limitait déjà aujourd'hui notre participation aux activités communes avec I'IDA.

Des pays qui ont à peu près la même dimension que la Suisse et les mêmes intérêts qu'elle dans leurs relations économiques internationales partagent au FMI et à la Banque mondiale un siège d'administrateur avec trois ou quatre autres pays. II y a ainsi un groupe qui comprend les pays nordiques (Danemark, Finlande, Islande, Norvège et Suède). Ces pays coopèrent étroitement pour exercer de façon efficace leur influence au sein de la Banque mondiale. Dans sa négociation avec les institutions de Bretton Woods, la Suisse a fait savoir qu'elle chercherait à obtenir un quota qui lui permettrait de s'assurer dans la pratique un siège permanent dans le Conseil des administrateurs de la Banque mondiale. Cette position dénote une volonté claire du Conseil fédéral de jouer un rôle majeur dans ces institutions.

On peut déduire de cette demande que le Conseil fédéral a l'intention de se donner les moyens nécessaires pour jouer un rôle actif au sein de la Banque. Ceci suppose que des ressources importantes soient consacrées à la définition

19. Voir à cet égard la réponse du Conseil fédéral à la question ordinaire Ulrich sur la collaboration avec l'IDA en cas d'adhésion à la Banque mondiale (90.1089). 
et à la réalisation des objectifs que la Suisse poursuivrait à partir de la position privilégiée qui serait la sienne. C'est donc peut-être sur la gestion de nos relations économiques extérieures et de notre coopération au développement qu'une adhésion poserait à la Suisse les plus grands défis. II s'agira le cas échéant pour le gouvernement suisse de se donner les moyens de ses ambitions.

\section{Indications blbllographiques}

- Message concernant la continuation de la coopération technique et de l'aide financière en faveur des pays en développement du 21 février 1990. - Berne, 1990. - $230 \mathrm{p}$.

- Message concernant la continuation du financement des mesures de politique économique et commerclale, y compris des mesures de désendettement, au titre de la coopération internationale au développement et un accord sur le statut juridique de la Société financière internationale en Suisse du 21 février 1990 - Berne 1990. $144 \mathrm{p}$.

- GERSTER, Richard. - Fallstricke der Verschuldung: Der Internationale Währungsfonds und die Entwicklungsländer. - Basel: Z-Verlag, 1982.

- HÜRNI, Bettina, "Der Beltritt der Schwelz zur Weltbank aus ökonomischer Sicht", in Schweizerische Zeitschrift für Volkswirtschaft und Statistik März 1980, p. 519.

- KAESER, Daniel, "Les Relations de la Sulsse avec les Institutions de Bretton Woods", in La Vie économique 2/89, p. 9-11, Berne.

- RICH, Georg; RUOSS, Eveline, "Les Contributions fournies par la Sulsse au FMI et à la Banque mondiale: ", in Vie économique 2/89, pp.12-14, Berne.

- VOELK, Karl . - Le Groupe de la Banque mondlale et la Sulsse. Union de Banques Suisses, 1987. - $74 \mathrm{p}$. 